\title{
Non-Thermal Plasma Ozone Generation
}

S. Pekárek

This paper reviews ozone properties, ozone applications and the mechanism of ozone production in non-thermal plasma. An analysis is made of the influence of a reduced electric field and discharge space temperature on ozone production. The phenomenon of discharge poisoning is also explained. Finally, a modern ozone production system based on dielectric barrier electrical discharge is described.

Keywords: ozone, non-thermal plasma, electrical discharge.

\section{Introduction}

In 1841 Christian Schonbein identified the characteristic odor of ozone in the vicinity of an electrical discharge and named it ozone after the Greek word ozein, meaning "smell". Ozone was identified as triatomic oxygen in 1867.

Ozone is a toxic gas. Its odor is detectable by most people at a level of $0.003 \mathrm{ppm}$ and becomes intolerable at concentrations about $0.15 \mathrm{ppm}$. Overexposure to ozone causes problems of the human respiratory system, watery eyes, coughing, heavy chest and a stuffy nose.

Ozone is an unstable gas, which begins to decompose instantly. Its half-life ranges from a few seconds to several hours. However the half-life decreases significantly in the presence of moisture, increased temperature and some metals - copper, silver, iron. Ozone decomposes to oxygen after being used, so no harmful byproducts result.

Ozone is a practically transparent gas with very strong absorption in the region of a wavelength of $245 \mathrm{~nm}$. Namely this property of ozone protects life on Earth from dangerous ultraviolet radiation emitted by Sun.

Ozone is a powerful disinfecting and oxidizing agent, and for this reason it is used in a wide range of applications such as treatment of municipal and waste water, food processing, fire restoration, restoration of buildings and other objects after floods, etc. When ozone comes into contact with organic compounds or bacteria, the extra atom of oxygen destroys the contaminant by oxidation. Thus ozone will neutralize virtually all organic odors, specifically those containing carbons as their base element. This will include all bacteria as well as smoke, decay and cooking odors. However, due its oxidizing nature ozone attacks and degrades natural latex rubber, thus for example a laminate silica gel should be applied to car and freezer door moldings.

Ozone is usually generated in one of three ways:

- Electrochemical generation - in this case an electric current passes through the liquid electrolyte and produces a mixture of gases, containing ozone.

- Generation of ozone by ultraviolet rays (this process takes place in the upper layers of the atmosphere).

- Generation of ozone in non-thermal plasma produced by electrical discharges.

Most of the ozone for practical applications is produced in non-thermal plasma generated by electrical discharges.

\section{Non-thermal plasma ozone generation}

Plasma is a quasi-neutral mixture of neutral and charged particles, having collective properties. It can be classified from several standpoints. One of these standpoints deals with the equilibrium between the temperatures of ions and electrons. When the temperatures of electrons and ions are the same we speak of thermal plasma. On the other hand, if the temperature of the electrons is higher than the temperature of the ions we speak about non-thermal plasma. The key advantage of non-thermal plasma is the "directed" energy consumption. In this case the energy delivered to the discharge is predominantly used for generating highly energetic electrons, and little energy is lost in heating the volume of a gas. Consequently, technologies based on non-thermal plasma can be highly effective in promoting oxidation, enhancing molecular dissociation or producing free radicals to stimulate plasmachemical reactions, which can be used for ozone generation or for other ecological applications such as decomposition of pollutants in air streams.

There are two main methods for producing non-thermal plasma:

- Acceleration of electrons by an electric field.

- Injection of electrons externally.

The first group of methods comprises different types of electrical discharges, and the second method involves the usage of an electron beam.

Due to the fact that the processes of ozone generation are carried out at atmospheric pressure, we shall focus on atmospheric pressure electrical discharges only. The main type of electrical discharge that can be used for the above-mentioned purpose is dielectric barrier discharge and corona discharge.

Dielectric barrier discharge is a general term for discharge that uses a dielectric material as a plasma stabilizer. The main types are:

- Silent discharge.

- Surface discharge.

The main feature of silent discharge is the dielectric layer, which covers at least one of the electrodes, sometimes both. Typically, materials with a high dielectric constant (ceramics or glass) are used. The most widely used silent discharge electrode configurations at the present time are parallel plate 
types or the wire-tube type. A common parallel plate electrode configuration for silent discharge is shown in Fig. 1.

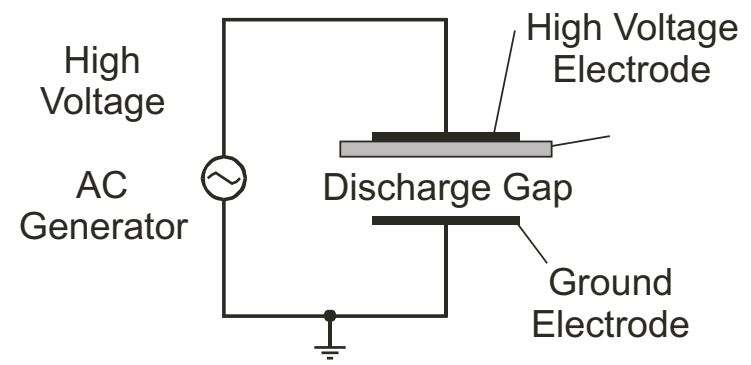

Fig. 1: Silent discharge electrode configuration

Surface discharge uses different types of design. The most frequent one is so-called packed bed discharge, which is shown in Fig. 2.

In this case, spherical pellets of dielectric material fill the discharge volume between two electrodes. If the voltage over the electrodes is increased, a weak glow appears at the surface

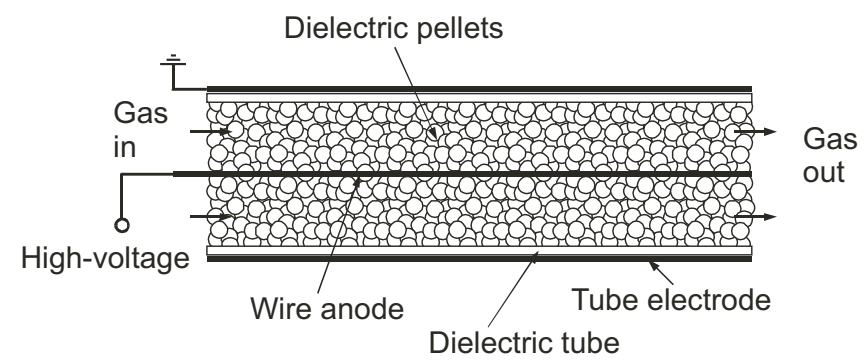

Fig. 2: Packed bed discharge electrode configuration

of the pellets. The pellets enhance the electric field in the contact regions among the adjacent pellets, and this leads to microbreakdowns in the gas. The materials often used are $\mathrm{BaTiO}_{3}, \mathrm{PbTiO}_{3}$ and others. A high voltage (about $15 \mathrm{kV}$ ) of frequency ranging from $50 \mathrm{~Hz}$ to about $10 \mathrm{kHz}$ is applied to generate the discharge.

Corona discharge is a relatively low power electrical discharge that takes place at or near atmospheric pressure. The corona is generated by a strong electric field, and at least one of the electrodes is a thin wire, a needle or has a sharp edge. The strong curvature is responsible for the high electric field, which is necessary for the ionization of neutral gas atoms. The other electrode is most frequently a plate or a cylinder.

Two ways are used to enhance the corona discharge power and therefore to increase its current-voltage range and at the same time to prevent the discharge transition to a spark:

- In the first case pulse voltage is applied to the electrodes [1].

- The second way of discharge stabilization involves the usage of the gas flow. In this case we talk about gas flow stabilized discharge or about atmospheric pressure glow discharge [2].

There are several types of electrode configurations of gas flow stabilized discharge. One of these involves the multi-needle to plate electrode configuration with the flow of a gas medium around the needle electrodes [3]. The disadvantage of this arrangement, which is shown in the top part of Fig. 3, is that to stabilize the discharge the velocity of the gas should be about 50 to $100 \mathrm{~m} / \mathrm{s}$.
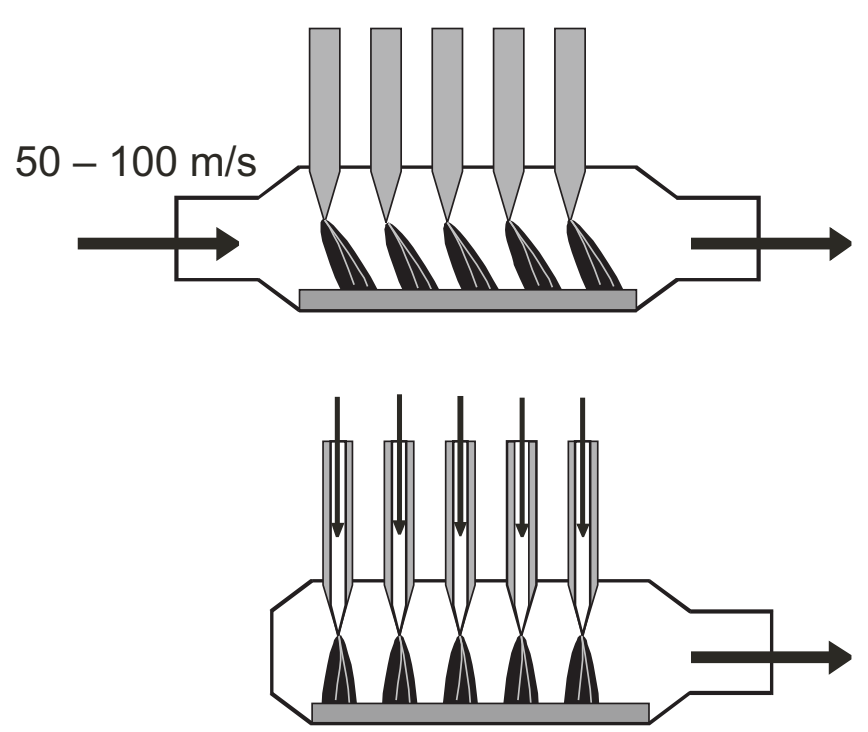

Fig. 3: Different modifications of the gas flow stabilized discharge

To relax the conditions applied to the gas velocity we have developed a new version of the discharge with a hollow needles to plate electrode configuration [4]. In this case the gas is supplied through the needles only - see botttom part of Fig. 3. The advantage of this arrangement is that all the gas medium passes through the discharge region and therefore it is affected by plasmachemical processes.

There are other types of electrical discharges (microwave discharge, capillary discharge, glidarc, etc.), which can be used for ozone generation. However, their application for these purposes is not so frequent.

The advantage of electrical discharges as a source of non-thermal plasma for ozone generation is that the plasma parameters can be relatively easily controlled by changing several variables, for example:

- The operating voltage of the discharge affects the magnitude of the electric field, and hence the energy of the charged particles in the plasma.

- The power controls the number of ionizations per unit volume per second and hence is an approximate control variable for the plasma density. It is also a possible control variable for the energy of the plasma constituents.

- The gas pressure controls the electron collision frequency and the mean free paths of all plasma constituents.

- The type of gas controls the ionization potential, and thus the energy required to produce an electron-ion pair in the plasma.

- The geometry of the electrodes can affect the energy input by altering the electric field, through changing the geometry of the anode-cathode configuration.

- Finally the cathode characteristics, such as the secondary emission coefficient, or the capability of thermoionic emission can also affect the discharge characteristics. 


\section{Mechanism of ozone generation}

There exist several models of ozone generation mechanisms, which differ mainly by the number of reactions involved [e.g. 5, 6, 7]. Generally speaking the mechanism of ozone generation by electrical discharge in air is rather complicated, due to the presence of nitrogen in air. This presence of nitrogen causes ozone destruction processes because of the existence of nitrogen oxides.

The role of the electrons produced in an electrical discharge is to excite and dissociate the oxygen and nitrogen molecules. The initial step in formation of $\mathrm{O}_{3}$ and $\mathrm{NO}_{x}$ is therefore the electron impact dissociation of molecular $\mathrm{O}_{2}$ and $\mathrm{N}_{2}$

$$
\begin{aligned}
& \mathrm{e}+\mathrm{O}_{2} \rightarrow \mathrm{O}+\mathrm{O}+\mathrm{e} \\
& \mathrm{e}+\mathrm{N}_{2} \rightarrow \mathrm{N}+\mathrm{N}+\mathrm{e}
\end{aligned}
$$

Atomic nitrogen reacts with $\mathrm{O}_{2}$ and $\mathrm{O}_{3}$ to form $\mathrm{NO}$ by the reactions

$$
\begin{aligned}
& \mathrm{N}+\mathrm{O}_{2} \rightarrow \mathrm{NO}+\mathrm{O} \\
& \mathrm{N}+\mathrm{O}_{3} \rightarrow \mathrm{NO}+\mathrm{O}_{2}
\end{aligned}
$$

$\mathrm{NO}$ and $\mathrm{NO}_{2}$ form a cycle for ozone destruction by the reactions

$$
\begin{aligned}
& \mathrm{O}+\mathrm{NO}_{2} \rightarrow \mathrm{NO}+\mathrm{O}_{2} \\
& \mathrm{NO}+\mathrm{O}_{3} \rightarrow \mathrm{NO}_{2}+\mathrm{O}_{2}
\end{aligned}
$$

$\mathrm{NO}_{2}$ is consumed by the following reactions

$$
\begin{aligned}
& \mathrm{NO}_{2}+\mathrm{O}_{3} \rightarrow \mathrm{NO}_{3}+\mathrm{O}_{2} \\
& \mathrm{NO}_{2}+\mathrm{NO}_{3} \rightarrow \mathrm{N}_{2} \mathrm{O}_{5}
\end{aligned}
$$

and regenerated by the reactions

$$
\begin{aligned}
& \mathrm{NO}+\mathrm{NO}_{3} \rightarrow \mathrm{NO}_{2}+\mathrm{NO}_{2} \\
& \mathrm{~N}_{2} \mathrm{O}_{5} \rightarrow \mathrm{NO}_{2}+\mathrm{NO}_{3} \\
& \mathrm{O}+\mathrm{N}_{2} \mathrm{O}_{5} \rightarrow \mathrm{NO}_{2}+\mathrm{NO}_{2}+\mathrm{O}_{2}
\end{aligned}
$$

The main ozone formation reaction dominant at high pressures is the reaction

$$
\mathrm{O}+\mathrm{O}_{2}+\mathrm{M} \rightarrow \mathrm{O}_{3}+\mathrm{M}
$$

An undesirable reaction, which determines the upper limit of $\mathrm{O}$ concentration is

$$
\mathrm{O}+\mathrm{O}+\mathrm{M} \rightarrow \mathrm{O}_{2}+\mathrm{M}
$$

In the case of a discharge in air, $\mathrm{M}$ represents molecular oxygen or nitrogen.

There are certainly other reactions that destroy ozone. One example of a catalytic reaction destroying ozone is

$$
\begin{aligned}
& \mathrm{NO}_{2}+\mathrm{O}_{3} \rightarrow \mathrm{NO}_{3}+\mathrm{O}_{2} \\
& \mathrm{O}+\mathrm{NO}_{3} \rightarrow \mathrm{O}_{2}+\mathrm{NO}_{2}
\end{aligned}
$$

This model is very simple, because it does not include reactions involving excited species, other molecules and other ozone self-destruction reactions. The reaction rates of reactions (1)-(14) depend on various parameters - for example on electric parameters of discharge, temperature, etc.

\subsection{Effect of electric field strength}

The dissociation rate coefficients of $\mathrm{O}_{2}$ and $\mathrm{N}_{2}$ by electron impact reactions (1) and (2) depend on the energy distribution of the electrons in the discharge. These coefficients are usually treated as functions of the reduced electric field, which is defined as the ratio of electric field strength per unit gas density $(E / n)$. A unit of the reduced electric field that is frequently used in the physics of electrical discharges, is 1 Townsend (abbreviation $1 \mathrm{Td}$ ), which is defined as $1 \mathrm{Td}=10^{-17} \mathrm{~V} \cdot \mathrm{cm}^{2}$. Following [5], the optimum reduced electric field for ozone formation from air is about $200 \mathrm{Td}$.

The mean energy of electrons as a function of the reduced electric field [8] obtained for streamer corona induced plasma in air at temperature $300 \mathrm{~K}$ is shown in Fig. 4. As can be seen, this mean energy increases with the applied electric field and for $(E / n)$ about $200 \mathrm{Td}$ reaches $5 \mathrm{eV}$.

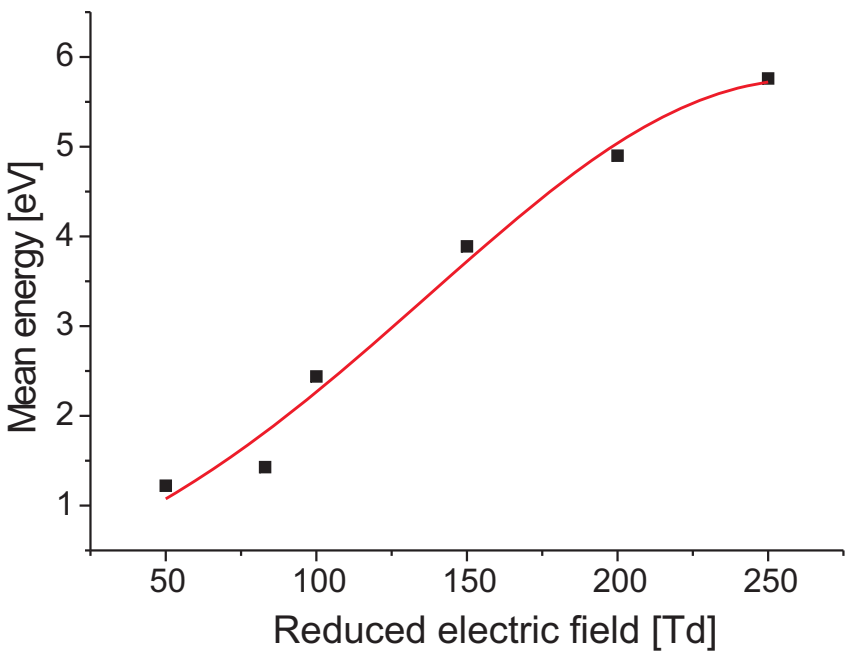

Fig. 4: Mean energy of electrons as a function of reduced electric field

\subsection{Effect of temperature}

Increasing gas temperature substantially reduces the ozone generation processes. The gas temperature $T_{\mathrm{g}}$, which appears in the rate coefficient of the reactions, is assumed to be the time and space averaged temperature in the discharge space. We have studied the dependence of the rotational temperature in the discharge space as a function of the discharge current [4]. It was found that as the discharge current increases the temperature in the discharge space also increases. For this reason, the quantities presented in Fig. 4 are given as a function of the discharge current.

The main ozone formation reaction dominant at atmospheric pressure is reaction (12), for which the reaction rate dependence on gas temperature $T_{\mathrm{g}}$ is given by the following expression

$$
k_{12}=2.5 \cdot 10^{-35} \exp \left(\frac{970}{T_{\mathrm{g}}}\right)\left[\mathrm{cm}^{6} \cdot \mathrm{s}^{-1}\right] .
$$

It is seen that the reaction rate of ozone generation decreases with increasing gas temperature. Thus if the temperature in the discharge space is increased from $300 \mathrm{~K}$ to 400 $\mathrm{K}$, then the reaction rate given by coefficient $k_{12}$ is 2.2 times decreased.

Unlike ozone, $\mathrm{NO}_{x}$ formation is favored by heat. Thus for example the ozone destruction reaction (6) is significantly enhanced by an increase in the gas temperature, or

$$
k_{6}=1.5 \cdot 10^{-12} \exp \left(-\frac{1300}{T_{\mathrm{g}}}\right)\left[\mathrm{cm}^{3} \cdot \mathrm{s}^{-1}\right] .
$$


If the temperature is increased from $300 \mathrm{~K}$ to $400 \mathrm{~K}$, then the reaction rate given by coefficient $k_{6}$ is more than 3 times increased.

The dependence of the reaction rates of ozone and nitrogen oxides generation on temperature causes an unpleasant effect, known as discharge poisoning - that is, the production of nitrogen oxides prevails over the production of ozone, so that finally no ozone is produced - see Fig. 5. This figure was obtained for a single hollow needle to plate discharge, with the airflow rate through the needle $5 \mathrm{slm}$. On the left and right vertical axes we can see the concentration of nitrogen oxides and ozone, respectively. The horizontal axis corresponds to the discharge current. Increasing the discharge current causes, for the same airflow rate, an increase of the temperature in the discharge volume.

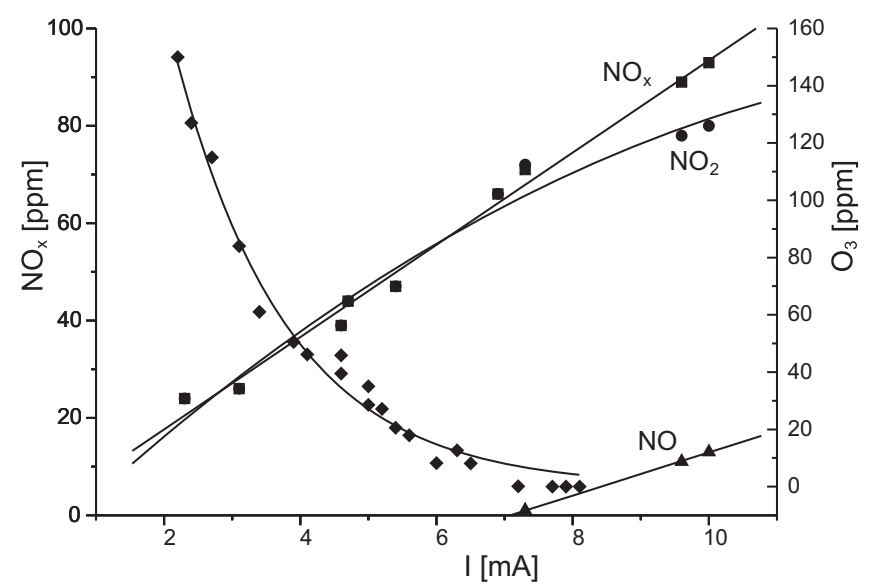

Fig. 5: Ozone and nitrogen oxides concentration as a function of current for a discharge with needle negative $(Q=5 \mathrm{slm})$

\section{Ozone generation system}

From the previous analysis it is clear that for efficient ozone production the electric discharge space must be kept both at a high pressure (atmospheric or even higher) and at a low temperature.

The first ozone production installation based on the electrical discharge for the purpose of water treatment went into operation in Nice in 1907. A typical present-day ozone generating system consists of two main components: an air preparation or oxygen production unit and an ozone non-thermal plasma production unit.

In commercial ozone generating systems air is more frequently used as a feed gas than oxygen (oxygen must be produced from air, which causes additional costs). Air preparation is critical for efficient ozone generation.

We studied the difference between ozone generation from air supplied by a compressor and ozone generation for the same condition from synthetic air. It was found [9] that the ozone production yield $-\mathrm{g}$ of ozone produced per $\mathrm{kW}-$ is higher for synthetic air. The reason is the presence of moisture and particulate matter in the air supplied by a compressor, which has a detrimental effect on ozone generation.

For the supply of air, either pressure fed systems or atmospheric pressure dryers are used. Pressure fed systems - i.e. systems with compressors - are more suitable than atmo- spheric pressure dryers, because they are able to provide (with filters, etc.) the necessary air quality in various climatic conditions. The air preparation unit also comprises rotameters, filters, regulating valves, etc.

An example of ozone production unit based on dielectric barrier discharge is shown in Fig. 6. The electrode arrangement makes use of a borosilicate glass tube 1-2 $\mathrm{m}$ in length and 20 to $50 \mathrm{~mm}$ in diameter. This tube is mounted inside a stainless steel tube to form a narrow annular discharge space. A conductive coating (for example a thin aluminium film) forms the high voltage electrode on the inside of a glass tube. The inner part of the stainless steel tube serves as a second electrode. Since the efficiency of ozone generation decreases strongly with rising temperature, modern ozonizers have narrow discharge gaps ranging from 1 to $3 \mathrm{~mm}$. The wall of the steel electrode is cooled by water. Large ozone generating units use several hundreds of tubes.

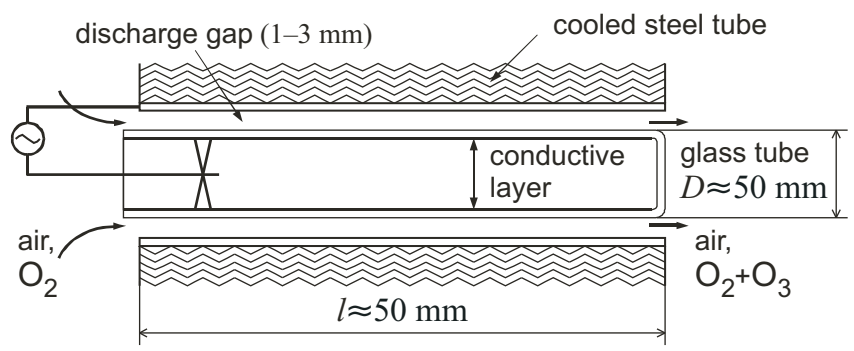

Fig. 6: Electrode arrangement of a dielectric barrier discharge for ozone production

\section{Conclusions}

Apart from small-scale production of ozone for medical purposes and for preparation of ultra-fine water, ozone is mainly prepared by non-thermal plasma technologies based on electrical discharges. Modern industrial ozone production systems are capable of producing several hundreds kilograms of ozone per hour at power consumption of several MW. Research in this field is oriented on progress toward higher attainable ozone concentrations and lower energy consumption i.e., toward higher ozone yields.

\section{Acknowledgement}

This work was supported by research program No: J04/98: 212300016 "Pollution Control and Monitoring of the Environment" of the Czech Technical University in Prague (sponsored by the Ministry of Education, Youth and Sports of the Czech Republic).

\section{References}

[1] Šimek, M., Člupek, M.: Efficiency of ozone production by pulsed positive corona discharge in synthetic air. J. Phys. D: Appl. Phys., 2002, Vol. 35, p. 1-5.

[2] Goosens O. et al: The DC glow discharge at atmospheric pressure. IEEE Trans. on Plasma Science, 2002, Vol. 30, No. 1, p.176-177.

[3] Napartovitch, A. P., Akishev Y. S.: DC glow discharge with fast gas flow for flue gas processing. In: Non Thermal 
Plasma Techniques for pollution control, NATO ASI Series, Springer-Verlag, 1992.

[4] Pekárek, S. et al: Hollow needle to plate electrical discharge at atmospheric pressure. Plasma Sources Sci. Technol., 1999, Vol. 8, p. 513-518.

[5] Kogelschatz, U., Eliasson, B.: Ozone generation from oxygen and air: Discharge physics and reaction mechanism. Ozone Science and Engineering, 1988, Vol. 10, p. 367-377.

[6] Kitayama, J., Kuzumoto, M.: Analysis of ozone generation from air in silent discharge. J. Phys. D: Appl. Phys., 1999, Vol. 32, p. 3032-3040.

[7] Mason, N. J., Skalny, J. D.: Experimental investigations and modelling studies of ozone producing corona discharges. Czech. J. Physics, 2002, Vol. 52, p. 85-94.

[8] McAdams, R.: Prospects of non-thermal atmospheric plasma for pollution abatement. J. Phys.D: Appl. Phys., 2001, Vol. 34, p. 2810-2821.
[9] Šimek, M., Člupek, M., Pekárek S.: Efficiency of ozone production in non-thermal plasma electrical discharges in synthetic air-comparative study. $16^{\text {th }}$ Int. Symp. on Plasma Chemistry, Taormina, Symp. Proc. Vol.II, 2003.

Prof. Ing. Stanislav Pekárek, CSc. phone: +420224352333

fax: +420224352331

e-mail:pekarek@feld.cvut.cz

Department of Physics

CTU in Prague

Faculty of Electrical Engineering

Technická 2

16627 Prague 6, Czech Republic 